\title{
Aprender ciencias experimentales mediante TIC en tiempos de covid-19: percepción del estudiantado
}

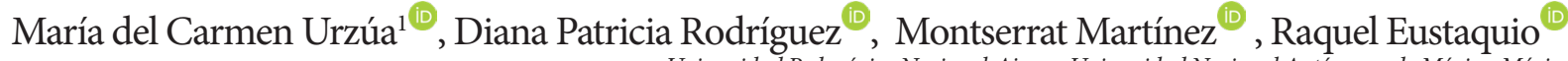 \\ Universidad Pedagógica Nacional-Ajusco, Universidad Nacional Autónoma de México, México
}

Autor de correspondencia: ${ }^{1}$ murzua@upn.mx

Recibido: 15 de julio de 2020 Revisado: 30 agosto de 2020 Aprobado: 12 de octubre de 2020 Publicado: 16 de noviembre de 2020

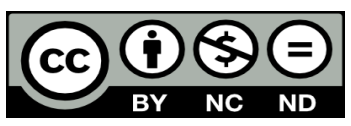

\section{Resumen}

Ante la pandemia por covid-19 pasamos inesperadamente a una educación remota de emergencia [ERE] mediada por el uso de TIC. En este contexto, desde el paradigma interpretativo, realizamos una investigación de carácter descriptivo para dar cuenta de las percepciones sobre el aprendizaje, de estudiantes universitarios del área de ciencias en una asignatura de carácter práctico - Laboratorio de Microbiología Experimental-, al pasar de una educación presencial a una ERE. Con un enfoque metodológico mixto, se diseñó y se validó un cuestionario con preguntas cerradas y abiertas, que fue resuelto por 56 estudiantes. Se utilizaron dos ejes analíticos: uno de carácter contextual y otro conceptual, para lo cual se realizó un análisis de contenido tanto cuantitativodescriptivo como cualitativocomprensivo. Los resultados muestran que más del $50 \%$ percibe que no se lograron los objetivos de la asignatura y que aprendieron menos. El 80 \% considera que la comunicación con los docentes fue buena. El espectro conceptual predominante del alumnado es tradicionalista. Por lo anterior, se concluye la importancia de trabajar con las TIC desde una perspectiva constructivista, en el marco de una "nueva visión de la ciencia".

Palabras clave: percepción, estudiante universitario, trabajos prácticos, TIC 


\title{
Learning experimental sciences through ICT in COVID-19 times: students' perception
}

\begin{abstract}
In the COVID-19 pandemic, we unexpectedly turned to an emergency remote education [ERE] mediated by ICT. In this context, from the interpretive paradigm, we conducted a descriptive research to report the perceptions about learning of university students of a science career, in a practical subject-Laboratory of Experimental Microbiology-having switched from a face-to-face education to an ERT. With a mixed methodological approach, we designed and validated a survey with closed and open questions that was solved by 56 students. Two analytical axes were used: a contextual one and a conceptual one, for which we carried out a content analysis, both descriptive-quantitative and comprehensive-qualitative. The results show that more than 50\% perceive that the objectives of the subject were not achieved and that they learned less. $80 \%$ consider that communication with teachers was good. The predominant conceptual spectrum of the student population is traditionalist. Therefore, we conclude the importance of working with ICTs from a constructivist perspective, within the framework of a "new vision of science".
\end{abstract}

Keywords: perception, university student, practical work, ICT

\section{Aprender ciências experimentais através das TIC em tempos de COVID-19: percepção dos estudantes}

\section{Resumo}

Confrontado com a pandemia COVID-19 inesperadamente recorremos a uma educação remota de emergência [ERE]. Neste contexto, a partir do paradigma interpretativo, realizamos uma investigação descritiva, para dar conta das percepções sobre a aprendizagem dos estudantes universitários da área de ciências em uma disciplina prática - Laboratório de Microbiologia Experimental -, ao passar de uma educação em sala de aula para uma ERE. Com uma abordagem metodológica mista, foi desenhado e validado um questionário com perguntas fechadas e abertas, que foi resolvido por 56 estudantes. Foram utilizados dois eixos analíticos: um de natureza contextual e outro conceptual, para o qual foi realizada uma análise de conteúdo quantitativo-descritivo e qualitativo-compreensivo. Os resultados mostram que mais de $50 \%$ percebem que os objetivos da matéria não foram alcançados e que aprenderam menos. $80 \%$ consideram que a comunicação com os professores foi boa. $\mathrm{O}$ espectro conceptual predominante dos estudantes é tradicionalista. Assim, conclui-se a importância de trabalhar as TIC numa perspectiva construtivista, no âmbito de uma "nova visão da ciência”.

Palavras-chave: percepção, estudante universitário, trabalho prático, TIC 
De acuerdo con Tamayo (2009), la didáctica de las ciencias dejó de ser durante la década de los ochenta

un conjunto de prescripciones curriculares cuya finalidad era trasladar la lógica de las disciplinas científicas a la escuela, para convertirse en una disciplina [...] cuya preocupación es la de describir, analizar y comprender los problemas más significativos en la enseñanza y aprendizaje de las ciencias. (p. 34)

Por lo anterior, para la didáctica de las ciencias, la reflexión epistemológica juega un papel fundamental (Adúriz-Bravo, 2008; Izquierdo, 2000). Así la naturaleza de la ciencia [NOS] — concepto propuesto por McComas y Olson (1998) - subyace a las investigaciones de concepciones sobre la ciencia (Lederman \& O’Malley, 1990; López-Mota et al., 2004; Mellado, 1996; Porlán, 1988; Rodríguez, 2007; Rowell \& Cawthron, 1982), concepciones sobre el aprendizaje de las ciencias (Marín \& Benarroch, 2010; Mellado, 1998; Porlán, 1988; Ravanal \& Quintanilla, 2012; Rodríguez \& LópezMota, 2004; Tsai, 2002) y concepciones de uso de TIC en el aprendizaje de las ciencias (Almirón \& Porro, 2014; Blancas \& Rodríguez, 2013; Urzúa \& Rodríguez; 2015). Vale la pena señalar que la mayoría de las investigaciones que relacionan las concepciones sobre ciencia, aprendizaje y uso de TIC se han realizado fundamentalmente desde la mirada del profesorado y en mayor medida en educación básica.

Actualmente, la enseñanza y el aprendizaje enfrentan un contexto particular, pues, ante la pandemia por covid-19, los sistemas educativos pasaron de una educación presencial a una educación remota de emergencia [ERE] mediada por las TIC, lo que ha originado una nueva línea de investigación educativa, interesada en el análisis y reflexión del impacto y repercusiones de este nuevo escenario en los procesos de enseñanza y de aprendizaje, como se aprecia en la tabla 1.

Tabla 1

Estudios sobre ERE

\begin{tabular}{ll}
\hline AUTOR (ES) & $\begin{array}{l}\text { ASPECTO DE INTERÉS U ORIENTACIÓN DE LA } \\
\text { INVESTIGACIÓN }\end{array}$ \\
\hline Beltrán et al. (2020) & $\begin{array}{l}\text { Reflexión acerca de la ERE desde una mirada sociológica contextual } \\
\text { Habib et al. (2020) }\end{array}$ \\
$\begin{array}{l}\text { Dificultades que han enfrentado los docentes durante el confinamiento } \\
\text { Propuesta de un modelo de evaluación global dirigido al nivel básico y al } \\
\text { superior }\end{array}$ \\
Sánchez et al. (2020) & $\begin{array}{l}\text { Investigación donde recuperan las problemáticas logísticas, tecnológicas, } \\
\text { pedagógicas y socio-afectivas que desafiaron profesores y alumnos desde } \\
\text { la perspectiva del docente } \\
\text { Abordan los efectos del confinamiento en el proceso de enseñanza y de } \\
\text { aprendizaje-identificados por docentes y alumnos-, a partir de la teoría } \\
\text { (2020) }\end{array}$ \\
\hline
\end{tabular}

Los antecedentes mostrados en la tabla 1 permiten entrever que hasta ahora las investigaciones se han dirigido a la educación como ámbito general, sin analizar los procesos educativos ni áreas disciplinares específicas. El enfoque se ha centrado en la percepción de los docentes, no del estudiantado, excepto pocos eventos que han promovido un análisis emergente de la visión del alumnado en torno a la ERE, como el caso del foro Aprender en la contingencia: las experiencias de estudiantes universitarios (Canal CODEIC/UNAM, 2020). 
Por lo cual hoy en día cobra relevancia indagar y conocer la mirada de los estudiantes universitarios de ciencias respecto a su percepción del proceso de aprendizaje durante la contingencia sanitaria por covid-19, particularmente en asignaturas de carácter práctico, cuyo lugar natural de trabajo es el laboratorio. Así, nos planteamos como pregunta de investigación: ¿cuáles son las percepciones del estudiantado respecto a su proceso de aprendizaje en una asignatura de ciencias naturales de carácter práctico -laboratorio- en la ERE mediada por TIC?

Con la intención de dar voz al alumnado en esta investigación, nos proponemos identificar el impacto que ha tenido este nuevo contexto ERE mediado por TIC en el proceso de aprendizaje del alumnado desde su visión o percepciones, en una asignatura de laboratorio.

\section{Metodología}

\section{Diseño de investigación}

Este estudio se realizó desde una perspectiva interpretativa (Ernest, 1995) con aproximación metodológica mixta, dado que el interés radica en tratar de dar sentido a las percepciones expresadas por el alumnado e interpretar su discurso, a partir de la descripción y comprensión de este.

Para ello, se diseñó un cuestionario en la plataforma Google formularios, basado en instrumentos anteriores de un equipo del grupo de investigación de Educación en Ciencias -EDUCIEN - de la Universidad Pedagógica Nacional de México, que trabaja la temática de concepciones epistemológicas, de aprendizaje y de uso de TIC.

El instrumento estaba conformado por 15 preguntas — cerradas y abiertas- distribuidas en tres secciones como se muestra en la tabla 2. En los cuestionamientos cerrados se solicitó argumentar la elección realizada para obtener respuestas que aportaran información suficiente para indagar las percepciones de los estudiantes respecto a los tópicos mencionados. Ello posibilitó un análisis de contenido tanto cuantitativodescriptivo como cualitativocomprensivo. Como el dato proviene de las respuestas del alumnado - que en la presente investigación se asume como su discurso-, en los resultados se incluyen algunas expresiones textuales dadas por ellos [E].

Con la finalidad de dar validez al instrumento diseñado y confiabilidad a la investigación, el cuestionario se piloteó con 21 personas de 17 a 26 años y se sometió a validación por jueceo, donde contamos con la colaboración de una psicóloga experta en el diseño de este tipo de instrumentos de investigación.

\section{Caracterización de los participantes}

La asignatura Microbiología Experimental se impartió en el semestre 2020-2 a 12 grupos de la Facultad de Química de la UNAM, que representa un total aproximado de 240 alumnos inscritos. El cuestionario fue contestado de manera voluntaria y anónima por 56 estudiantes inscritos en cuatro grupos de clase.

Los cuatro grupos participantes en esta investigación utilizaron la plataforma Moodle antes y durante el contexto ERE. Sin embargo, su uso no cambió, pues en ambos momentos se utilizó para evaluación y como repositorio de información. Los profesores de estos cuatro 
grupos utilizaron la colección de videos Técnicas básicas de microbiología, desarrollada y validada por docentes de la asignatura (Urzúa, 2019) antes de la pandemia, para la explicación de las técnicas previa a su realización en el laboratorio, y durante el contexto ERE, con el fin de que conocieran tales procedimientos.

\section{Tabla 2}

Estructura del instrumento de investigación

\begin{tabular}{|c|c|c|}
\hline SECCIÓN & $\begin{array}{l}\text { Número de } \\
\text { preguntas }\end{array}$ & INFORMACIÓN RECABADA \\
\hline 1 & 4 & $\begin{array}{l}\text { Datos generales sobre la asignatura, el tipo de conexión y dispositivos } \\
\text { electrónicos con los que cuentan para realizar las actividades escolares en casa, } \\
\text { y si cuentan o no con un espacio exclusivo para efectuar su trabajo académico; } \\
\text { todo lo anterior, durante el contexto de ERE. }\end{array}$ \\
\hline 2 & 4 & $\begin{array}{l}\text { Conocimiento y tipo de uso que los alumnos le daban a las TIC antes de la } \\
\text { contingencia y durante el trabajo de ERE, ya fuera para fines recreativos o } \\
\text { académicos por iniciativa propia y/o por petición del profesor. }\end{array}$ \\
\hline 3 & 7 & $\begin{array}{l}\text { Tipo de interacción entre alumno y profesor durante las clases no presenciales, } \\
\text { el logro o no de los objetivos de la asignatura, la comunicación — sincrónica y } \\
\text { asincrónica - y la evaluación de la asignatura. }\end{array}$ \\
\hline
\end{tabular}

\section{Contexto de la asignatura}

La asignatura de carácter práctico Microbiología Experimental se imparte en el quinto semestre de dos carreras de la Facultad de Química de la UNAM. Tiene como antecedente Microbiología General, en la que se revisan los contenidos teóricos que sirven de base a esta asignatura. Se imparte en laboratorio seis horas por semana, distribuidas en dos sesiones de tres horas cada una y tiene como objetivos:

Aplicar correctamente las normas de higiene y bioseguridad en el laboratorio de microbiología. Desarrollar correctamente las técnicas básicas para el estudio de los microorganismos. Discriminar las condiciones nutricionales y de cultivo para los diferentes grupos microbianos. Evaluar el efecto de los agentes físicos y químicos en el crecimiento microbiano. Caracterizar a las eubacterias en función de su capacidad metabólica. Aplicar diferentes métodos de cuantificación de microorganismos en diversos productos (Facultad de Química-UNAM, julio de 2020).

Como se aprecia, los objetivos de la asignatura denotan un enfoque positivista de la ciencia, pues remiten únicamente al desarrollo de habilidades técnicas y dejan de lado el fomento de habilidades de análisis y reflexión sobre los fenómenos, es decir la comprensión de ellos como parte fundamental del aprendizaje, visto desde un enfoque constructivista (Rodrigo \& Cubero, 2000), donde no hay verdades absolutas que 'aprehender' (Adúriz-Bravo, 2008).

Dinámica de trabajo. Ante la libertad de cátedra que existe en la UNAM para impartir la docencia, los docentes utilizan diferentes estrategias y recursos que apoyen su enseñanza. Sin embargo, de manera general, las dinámicas de trabajo seguidas de manera presencial y 
en contexto ERE se presentan en la tabla 3.

Tabla 3

Comparación de la dinámica de trabajo en la sesiones presenciales y ERE

\begin{tabular}{|c|c|c|}
\hline $\begin{array}{l}\text { Tipo de } \\
\text { sesión }\end{array}$ & Secuencia de actividades & TIC y uso \\
\hline Presenci & $\begin{array}{l}\text { IInicio: solicitud de material, explicación de la técnica y } \\
\text { resolución de dudas sobre la misma, Desarrollo: } \\
\text { ejecución de técnicas o revisión de resultados } \\
\text { Fin: entrega de material } \\
\text { En algunas sesiones, se efectúa la evaluación. } \\
\text { (Urzúa, 2019; Urzúa \& Rodríguez, 2017) }\end{array}$ & $\begin{array}{l}\text { Herramientas ofimáticas, Facebook, blogs, } \\
\text { simuladores, Moodle, videos, buscadores y } \\
\text { bases de datos, correo electrónico y tutoriales, } \\
\text { las que en la mayoría de los casos son usadas } \\
\text { desde un enfoque técnico transmisor que } \\
\text { implica la búsqueda y consulta de } \\
\text { información, para su posterior transmisión a } \\
\text { los estudiantes (Urzúa \& Rodríguez, 2016). }\end{array}$ \\
\hline ERE & $\begin{array}{l}\text { Varió entre los grupos según el tema a revisar. Sin } \\
\text { embargo, algunas actividades fueron constantes y su } \\
\text { secuencia corresponde a: Inicio: examen previo, } \\
\text { presentación del tema y resolución de dudas sobre los } \\
\text { videos de las técnicas, } \\
\text { Desarrollo: presentación de ejemplos de aplicación de } \\
\text { las técnicas en diferentes ámbitos y planteamiento del } \\
\text { reporte de la práctica — que pudo ser la elaboración de } \\
\text { un proyecto o bien la interpretación de resultados } \\
\text { obtenidos en semestres anteriores y que los docentes } \\
\text { conservaban-. } \\
\text { Fin: Acuerdos para la siguiente sesión }\end{array}$ & $\begin{array}{l}\text { Además de las utilizadas en sesiones } \\
\text { presenciales, se agregaron las relacionadas } \\
\text { con videoconferencias para la comunicación } \\
\text { sincrónica con los estudiantes. }\end{array}$ \\
\hline
\end{tabular}

\section{Marco de interpretación}

Para analizar las percepciones del alumnado en torno a su aprendizaje de Microbiología Experimental al pasar de la educación presencial a la ERE mediada por TIC, utilizamos dos ejes de análisis: uno contextual, que identifica el impacto generado por la emergencia sanitaria; y otro conceptual, que distingue los espectros conceptuales de los estudiantes. Para ello, se realizó un análisis del contenido tanto cuantitativo-descriptivo como cualitativocomprensivo, dado que "explora las relaciones entre los textos y la realidad haciendo visibles los discursos" (Urra et al., 2013, p. 50).

A continuación, se describen los dos ejes mencionados y sus correspondientes categorías de análisis:

Eje contextual: da cuenta del impacto que ha tenido el contexto de la pandemia en el proceso de aprendizaje. Está formado por 4 categorías de análisis que emergieron del análisis de discurso realizado a las respuestas dadas por los alumnos a 4 de las 7 preguntas de la tercera sección del cuestionario, que estaban encaminadas a la reflexión sobre el desarrollo del aprendizaje de una materia experimental durante el confinamiento y del antecedente de trabajo de Sánchez et al. (2020). Estos resultados son tratados cuantitativa y cualitativamente.

Eje conceptual: para interpretar lo expresado por el alumnado respecto a cómo perciben que fue su aprendizaje en Microbiología Experimental, aludimos al marco categorial de espectros conceptuales (Urzúa \& Rodríguez, 2015) que engloban las concepciones epistemológicas, de aprendizaje y uso de TIC a partir de la idea planteada por Rodríguez (2007), de que las concepciones sobre ciencia y aprendizaje pueden interpretarse desde tres 
enfoques teóricos de referencia y, por ende, que las concepciones sobre uso de TIC también pueden enmarcarse en tres enfoques (Blancas \& Rodríguez, 2013). Esto da lugar a tres tipos de espectros conceptuales integrados por un perfil epistemológico, uno de aprendizaje y uno de uso de TIC (tabla 4). A continuación, se presenta una breve descripción de cada espectro conceptual:

Tradicionalista. Integrado por los perfiles empirista-positivista, asociacionista y transmisivo-reproductor. Los alumnos tienen una visión tradicional de la ciencia, enmarcada por el método científico, un aprendizaje de reproducción de conocimientos y, un uso de TIC transmisivore-productor que les permite memorizar la información recibida del docente.

Pragmatista. Integrado por los perfiles epistemológicos racionalistas. El alumnado tiene una visión de la ciencia como producto de la mente humana, que apunta a la lógica y al razonamiento; un perfil de aprendizaje cognoscitivista, de acomodación e integración de conocimientos; y uno práctico-integrador que guía un uso de TIC de practicidad que les permite complementar y organizar información.

Constructivista. Integrado por el perfil epistemológico constructivista de la ciencia que implica la construcción y elaboración de nuevos conocimientos a través de modelos; perfil constructivista donde el aprendizaje es visto como un proceso de construcción del alumno al interactuar con sus pares y con las herramientas a su alcance, en este caso, las TIC, a las que le da un uso crítico para proponer y aplicar su conocimiento, correspondiente al perfil transformadorconstructor social.

Tabla 4

Espectros conceptuales integrados por los perfiles epistemológico, de aprendizaje y uso de TIC

\begin{tabular}{cccc}
\hline Espectros & Perfil epistemológico & Perfil de aprendizaje & Perfil de uso de TIC \\
\hline Tradicionalista & Empirista-positivista & Asociacionista & Transmisivo-reproductor \\
Pragmatista & Racionalista & Cognoscitivista & $\begin{array}{c}\text { Práctico-integrador } \\
\text { Constructivista }\end{array}$ \\
Constructivista & Constructivista & $\begin{array}{c}\text { Transformador-constructor } \\
\text { social }\end{array}$ \\
\hline
\end{tabular}

Tomado de "Espectros conceptuales — epistemológico, cognitivo y de uso de TIC— de estudiantes de bachillerato tecnológico en la clase de biología” de Urzúa, et al., 2019, Bio-Grafía. Escritos sobre la Biología y su Enseñanza, 12(23), p.136. https://doi.org/10.17227/bio-grafia.vol.12.num23-9355.

De esta forma, en las respuestas de los estudiantes se buscaron rasgos que dieran pistas de su percepción sobre el aprendizaje, y se interpretaron sus argumentos desde los espectros conceptuales. En las expresiones textuales dadas por los alumnos, transcritas en el apartado de resultados de este eje, se subrayan algunas palabras o frases que indican la correspondencia con dicho espectro conceptual.

\section{Resultados}

Para contextualizar el impacto del contexto ERE en el aprendizaje de los estudiantes, presentaremos las condiciones y herramientas de trabajo que utilizaron los estudiantes, 
además de comparar las TIC utilizadas por los alumnos antes y durante ERE, información que se solicitó al inicio del cuestionario en la sección Datos generales.

\section{Elementos de las condiciones de trabajo de los estudiantes en el contexto ERE}

Ante el contexto de emergencia sanitaria por covid-19, un aspecto indagado en los participantes se refiere a la disponibilidad de un espacio exclusivo para desarrollar su actividad académica, en virtud de que se considera un factor que puede influir en el aprendizaje. La información que permite evidenciarlo procede de dos preguntas de la primera sección del cuestionario. En la gráfica 1 se aprecia que más de la mitad del estudiantado cuenta con un espacio exclusivo para el trabajo académico, siendo en el 86 \% de los casos su habitación.

\section{Gráfica 1}

Distribución del espacio de trabajo exclusivo

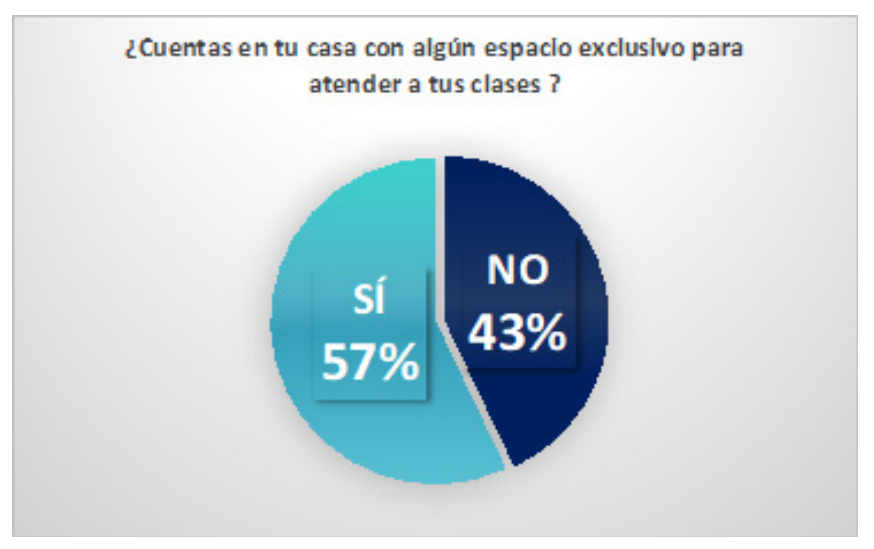

Este factor es importante en el presente estudio, puesto que alrededor del $10 \%$ del estudiantado siente afectado su proceso de aprendizaje al no contar con un espacio propio como se aprecia a continuación:

- E1: porque existen muchos más distractores que en la forma presencial lo que hace más difícil el aprendizaje.

- E2: me cuesta trabajo concentrarme en clases en línea.

- E3: es difícil poder concentrarse en casa.

- E4: no es el mismo aprendizaje que al estar en un salón de clases.

Datos comparativos sobre las TIC utilizadas por los alumnos, 'con fines académicos', antes y durante la emergencia sanitaria

Otro aspecto contextual indagado estuvo en torno al uso de las TIC con fines académicos antes y durante la contingencia por covid-19, por iniciativa propia y por petición del profesor. En el cuestionario les fueron presentadas 28 TIC: Word, Excel, Publisher, PPT, Facebook, Twitter, Instagram, correo electrónico, WhatsApp, YouTube, EcuRed, Moodle, Edmodo, Blackboard, blogs educativos, tutoriales, simuladores, wikis, programas para hacer mapas conceptuales y mentales, bases de datos - TESIUNAM, INEGI, etc.-, bibliotecas digitales, editores de páginas web, foro de debate, PLE, podcast, programas para elaborar líneas del tiempo, Prezi y software educativo.

Aquí retomamos solo las respuestas relativas al uso de TIC con fines académicos y 
presentamos los resultados cuantitativos. La suma de los datos no representa el total de alumnos, sino el de las respuestas dadas. En esta sección del cuestionario se podía elegir más de una opción de respuesta. La gráfica 2 muestra que antes del confinamiento y por iniciativa propia, el estudiantado utilizaba más aquellas TIC que les permitían procesar textos, buscar o presentar información, comunicarse entre compañeros y con el profesor, así como videos y tutoriales de apoyo para su aprendizaje y plataformas online.

\section{Gráfica 2}

TIC más utilizadas académicamente antes de ERE

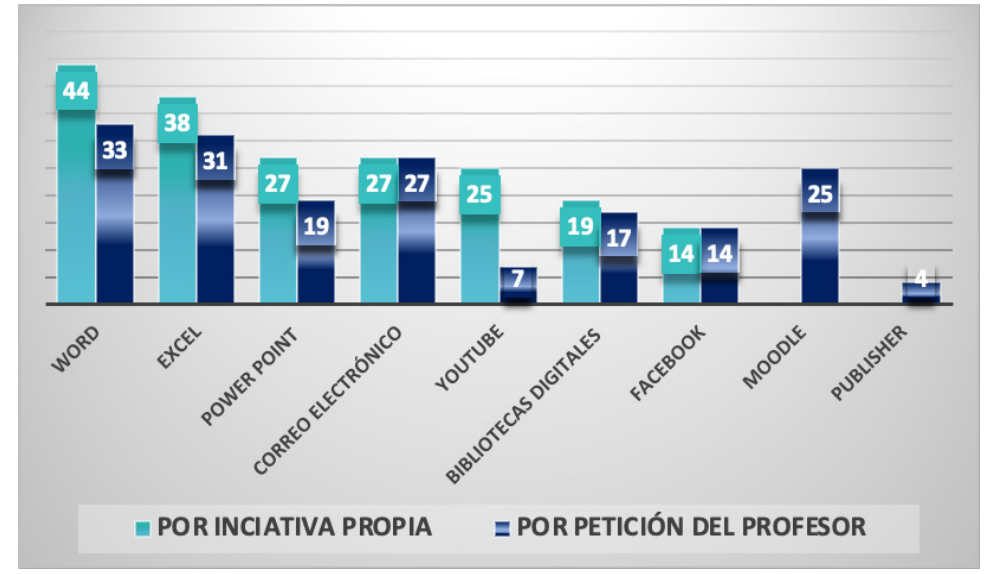

Además, los resultados muestran que durante ERE no hubo un cambio significativo en el uso de TIC para el aprendizaje de Microbiología Experimental, lo que causó, como veremos más adelante, que los estudiantes sintieran la falta de herramientas que les permitieran aprender la parte experimental de la materia como, por ejemplo, el uso de simuladores o softwares educativos.

\section{Análisis del eje contextual}

Las 4 categorías que guiaron el análisis de este eje son: logro de objetivos, comparación del aprendizaje en clases presenciales vs. no presenciales, comparación de la forma de evaluar en las clases presenciales vs. no presenciales y comunicación mediada por el uso de las TIC. A continuación, presentamos los resultados obtenidos para cada categoría.

\section{Logro de objetivos}

Respecto a la percepción de los estudiantes sobre el cumplimiento de los objetivos, preguntamos: ¿Consideras que, en las clases no presenciales, con el uso de las TIC, lograste alcanzar los objetivos planteados en el programa de la asignatura? Las opciones de respuesta fueron sí y no. En la gráfica 3 se aprecia que no hay gran diferencia entre el número de estudiantes que percibió que no se lograron los objetivos de la asignatura y los que sí lo percibieron.

Como los objetivos de la asignatura hacen referencia a la correcta aplicación de las técnicas básicas para estudiar a los microorganismos, su percepción es que no se lograron los objetivos, como se visualiza a continuación:

- E5: se necesita la parte experimental para lograr los objetivos del curso.

- E6: al ser una materia experimental y tomar clases en línea, no se logra adquirir 
las habilidades de trabajo experimental que se realiza en el laboratorio.

- E7: porque es una materia experimental, lo que debes hacer es la práctica. Para

lo teórico se llevó una asignatura previa.

\section{Gráfica 3}

Porcentaje de respuestas sobre el logro de los objetivos en ERE

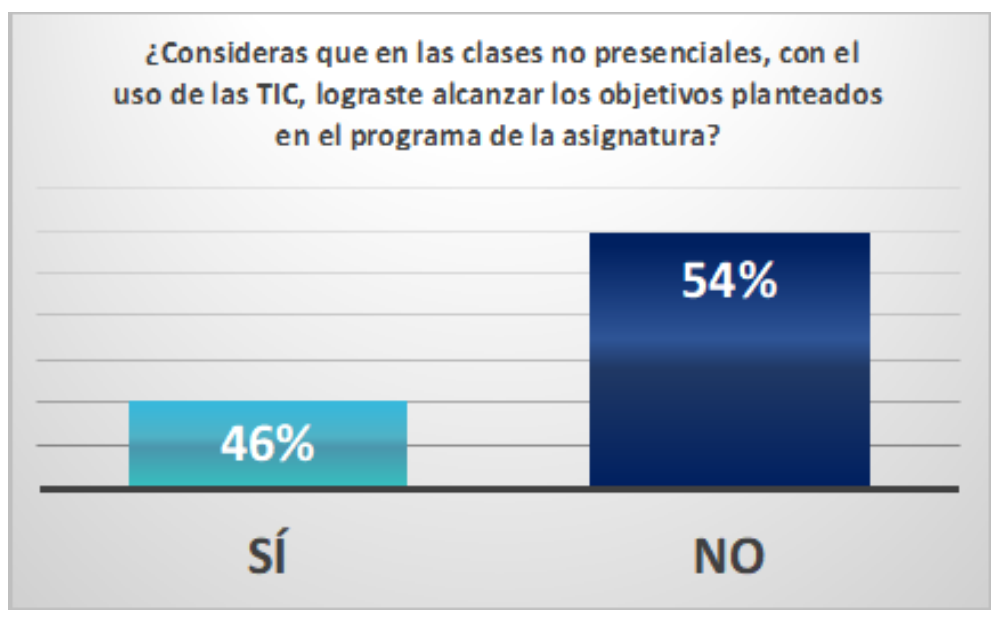

\section{Comparación del aprendizaje en clases presenciales $v$ s. no presenciales}

Ante la pregunta: ¿Consideras que el aprendizaje en las clases NO presenciales, que se han desarrollado con el uso de las TIC, en comparación con el aprendizaje de las clases presenciales, ha sido? con opciones de respuesta mayor, menor o igual, en la gráfica 4 se aprecia que más de la mitad de los estudiantes perciben que aprendieron menos durante la ERE en comparación con las clases presenciales. Solo una mínima parte considera que su aprendizaje fue mayor.

\section{Gráfica 4}

Distribución de respuestas respecto a la comparación de su aprendizaje

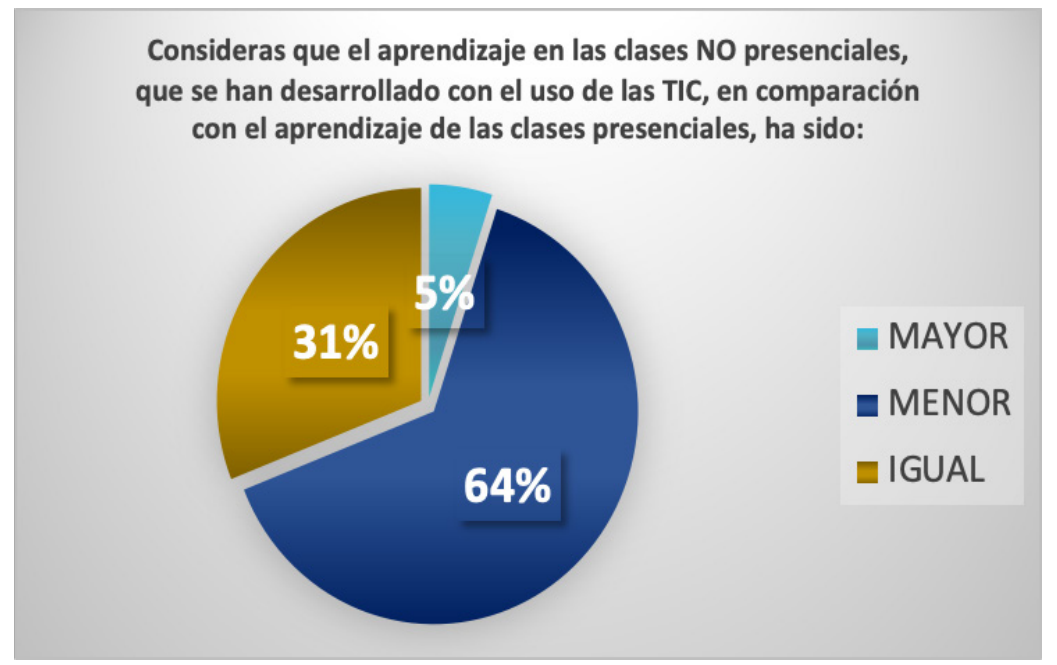


Entre los comentarios de los estudiantes que perciben haber aprendido más, algunos hacen referencia a procesos cognitivos superiores como el siguiente caso:

- E8: la parte teórica permanece igual, la parte práctica no. Sin embargo, la intención al momento de realizar informes fue razonar resultados experimentales - de artículos-. En mi opinión, los procedimientos de las prácticas no realizadas no son tan complicados, así que lo importante es la interpretación de los mismos y sus resultados.

En tanto, quienes perciben haber tenido un menor aprendizaje destacan la ausencia del trabajo manual como se aprecia en los siguientes comentarios:

- E9: porque al ser una materia experimental y tomar clases en línea no se logra adquirir las habilidades de trabajo experimental que se realiza en el laboratorio.

- E10: lo que más perjudica fue la parte experimental, en relación con las habilidades/destrezas manuales que estas nos proporcionan.

\section{Comparación de la forma de evaluar en clases presenciales y ERE}

Un aspecto fundamental para conocer el aprendizaje logrado por los estudiantes tiene que ver con la evaluación, por tanto, se les preguntó: ¿Consideras que la forma de evaluar de tu profesor en las clases NO presenciales que se han desarrollado con el uso de las TIC, en comparación con la evaluación durante las clases presenciales, ha sido? con opciones de respuesta igual o diferente. La gráfica 5 muestra que la mayoría de los participantes percibió que la evaluación fue diferente y los argumentos se relacionan con la ausencia de trabajo práctico y el no adquirir las habilidades manuales propias de la asignatura, como, por ejemplo:

- E11: porque en laboratorio hay bitácora, exámenes previos, exámenes de bloque, exámenes prácticos de manejo del material, etc.

- E12: porque en el caso de laboratorios no hacíamos trabajo práctico, sino es igual.

- E13: porque se mandan más trabajos y se evalúa todo menos lo práctico.

\section{Gráfica 5}

Visión de los estudiantes respecto a la forma de evaluación

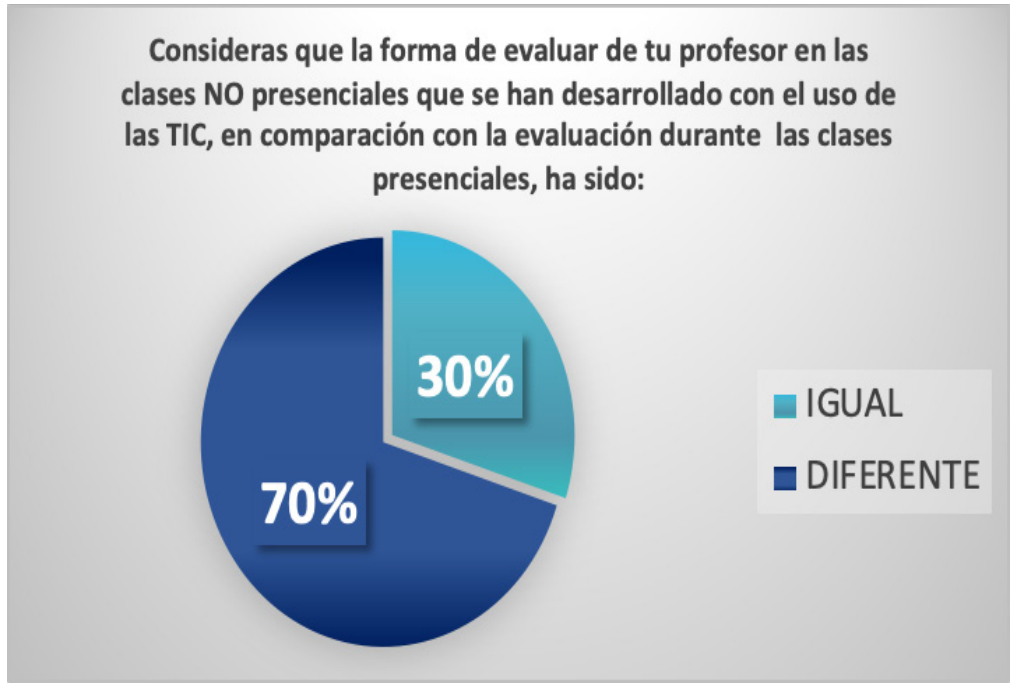




\section{Impacto de la comunicación}

El análisis de este aspecto se dividió en 2 subcategorías, la primera relativa al nivel de comunicación percibido por el alumnado y la segunda relacionada con la utilidad de la comunicación asincrónica, posibilitada por el uso de las TIC.

Para indagar el nivel de comunicación entre estudiante y docente, se planteó la pregunta: En este contexto de contingencia, donde la comunicación está mediada por las TIC, ¿cómo ha sido tu interacción con los profesores de tu asignatura? Hubo 5 opciones de respuesta, que van de muy mala a muy buena. Los resultados se muestran en la gráfica 6 , donde la mayoría de los estudiantes menciona que la comunicación con el docente fue buena o muy buena:

- E14: se puede estar en comunicación con los profesores en cualquier momento, así como el material de trabajo y las tareas que se entregan. Considero que ayudan a tener una buena comunicación por lo que ayuda en las materias.

- E15: se me facilitó conseguir información y comunicación con los profesores.

En esta subcategoría, quienes percibieron que la comunicación fue mala o muy mala, mencionan que:

- E16: no hay una buena comunicación con los profesores.

- E17: no tenemos la misma confianza de preguntar o no hay la misma disposición.

- E18: siguen existiendo problemas de comunicación entre alumnos y profesores.

\section{Gráfica 6}

Visión de los estudiantes respecto al nivel de comunicación con sus profesores

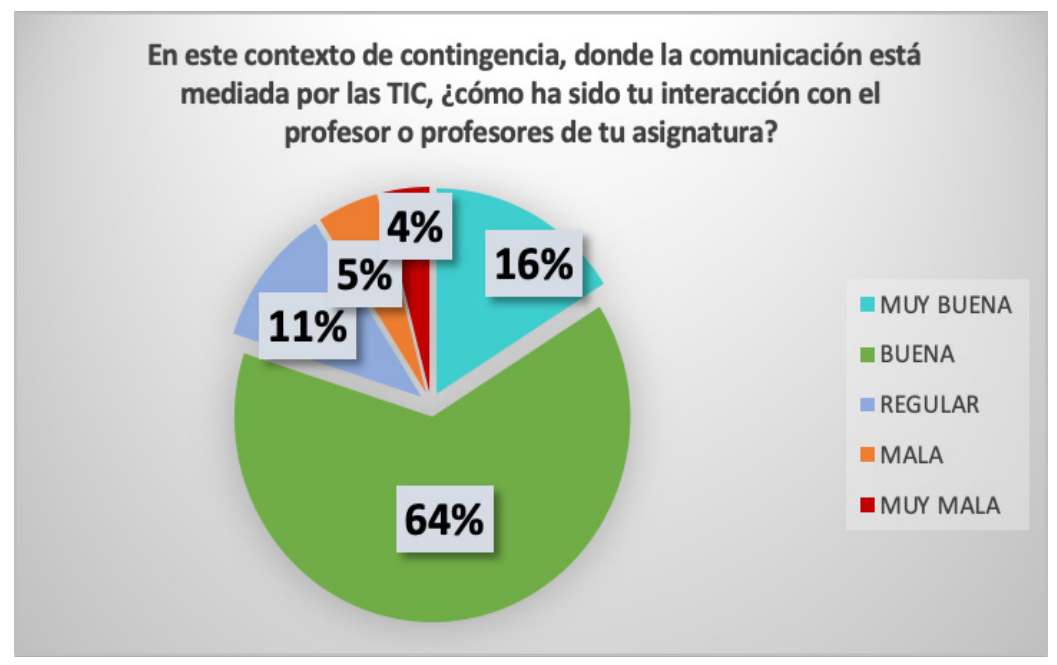

Respecto a la subcategoría utilidad de la comunicación asincrónica, se planteó la pregunta: En general el uso de las TIC permite la comunicación y el acceso al material de trabajo y de consulta tanto en tiempo real como en momentos diferentes a la clase. ¿Esto te ha sido útil durante este contexto de contingencia sanitaria? Las opciones de respuesta fueron sí o no. En la gráfica 7 se aprecia que prácticamente todos los estudiantes indican que fue útil la comunicación asincrónica. 


\section{Gráfica 7}

Visión de los estudiantes sobre la utilidad de la comunicación asincrónica

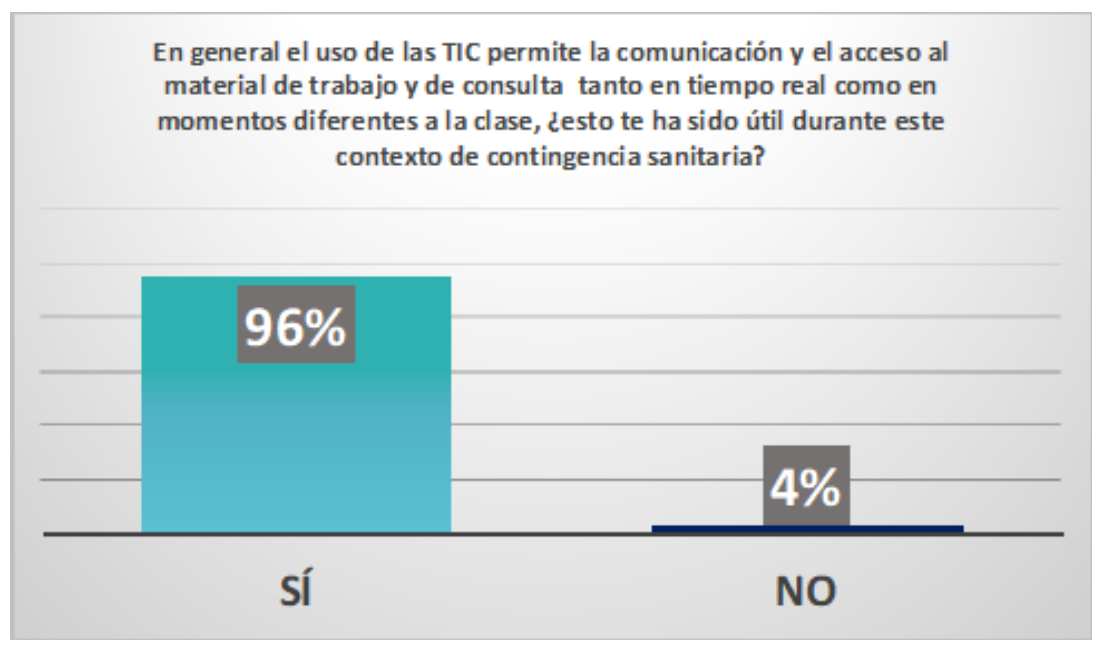

Algunos aspectos que denotan las ventajas que ellos observaron en la comunicación asincrónica son:

a) Consultar las clases grabadas. Si por alguna circunstancia no pudieron conectarse al momento de la clase, cuentan con los contenidos impartidos por el profesor - lo que no sucede en un ambiente presencial-. En sus propias palabras:

- E19: para estudiar o recapitular lo visto, para la realización de tareas es de mucha utilidad tener el material visto para tener información más completa.

b) La posibilidad de ver nuevamente el material y consultarlo en el momento en que lo requieran:

- E20: porque el material lo podemos consultar cuando sea necesario.

c) Aprender a su propio ritmo y de acuerdo con sus necesidades al concebir las TIC en asincronía como apoyo:

E21: en caso de que se olvide o sea necesario más información respecto al tema.

Por otro lado, esta ERE originada por covid-19, evidenció la importancia que cobran los aspectos socioemocionales, como lo plantean Mellado y Blanco (2012), "lo cognitivo configura lo afectivo y lo afectivo lo cognitivo" (p. VII), y fue expresado así por los alumnos:

- E22: debido a la situación de la pandemia, en lo personal me sentía con ansiedad todo el tiempo y pensaba en todo menos en la escuela.

- E23: ninguno de los profesores sabía cómo tomar esta emergencia. No había algún protocolo. En su mayoría, las clases fueron improvisadas o no se daban en tiempo y forma. En otras, simplemente no hubo contacto alguno. Además, que hay que resaltar que estábamos en riesgo sanitario, donde muchos compañeros e incluso sus familias pueden estar afectadas y eso no les da libertad de atender las clases. Sin contar que muchos no tenemos la infraestructura para tomarlas. 
Además, reconocieron como una virtud la comprensión, flexibilidad y accesibilidad de los docentes al momento de evaluar:

- E24: considero que ambos son comprensibles y en este caso son justos.

- E25: se han portado más accesibles en algunas materias.

- E26: algunos han tenido más flexibilidad al momento de calificar.

- E27: porque toman en consideración que cada persona vive una situación diferente ante la contingencia.

\section{Análisis del eje conceptual}

Presentamos el análisis de los argumentos proporcionados por los estudiantes, a partir del marco analítico de los espectros conceptuales. En sus respuestas se buscaron rasgos que dieran cuenta de la presencia de dichos espectros - espectros (tabla 4), para estudiar la congruencia y coherencia entre los perfiles epistemológicos, de aprendizaje y de uso de TIC.

Al leer detalladamente los argumentos de las respuestas del alumnado a las preguntas indicadas en el eje contextual y al realizar el análisis de su discurso, encontramos que el espectro pragmático no está presente en las percepciones del estudiantado, pues solamente hubo algunos esbozos, que no fueron consistentes en ningún estudiante, y dado que tampoco se evidenció su presencia en las opiniones dadas, por lo que no ahondaremos en este espectro.

A continuación, citamos algunos ejemplos que aluden al espectro constructivista, aunque solo el $5 \%$ de los argumentos de los estudiantes se refieren a dicho ámbito:

- E28: en mi opinión, los procedimientos de las prácticas no realizadas no son tan complicados, así que lo importante es la interpretación de los mismos y sus resultados.

Aquí, aunque se hace alusión a la falta de práctica, se resalta que lo importante es razonar e interpretar los resultados, lo que implica habilidades cognitivas para analizar y comprender la información que de forma autodidacta buscan, para lograr después interpretar los resultados no obtenidos experimentalmente por ellos.

En el siguiente comentario, un alumno declara el valor de una actividad realizada en la materia, que requirió un rol activo al indagar y construir preguntas previas a la clase, de manera que el alumno tuviera conocimientos previos que le sirvieran para construir su conocimiento sin que la responsabilidad de este proceso recayera en el profesor:

- E29: la clase fue muy buena. Me agradó mucho cuando se dividía en dos la clase, teniendo que contestar algunas cosas y luego se contestaban dudas y se veía a detalle el tema. Creo que eso ayudaba a que pusieras atención y estudiaras.

Algunos estudiantes reconocen que el aprendizaje bajo ERE fomentó un papel activo en su proceso de aprendizaje, como se aprecia a continuación:

- E30: aprendí a ser autodidacta.

- E31: porque uno al ser autodidacta, trata de entender y al entenderlo se te queda grabado.

- E32: quedaron muchas lagunas, que si bien al ser temas nuevos es más difícil poder 
dominarlos de forma autónoma.

- E33: porque he aprendido mucho leyendo artículos sobre las dudas que tengo.

El estudiantado busca respuestas por iniciativa propia, de tal forma que las TIC sirven como fuente de información para resolver sus dudas. Hacerlo de forma autónoma le permite profundizar en sus conocimientos y hacer una construcción propia de los mismos, respecto a lo cual Martin y Marchesi (citados por Medina et al., 2020) señalan que las TIC permiten, entre otras potencialidades, el desarrollo de la autonomía de gestión de conocimientos y la co-construcción de los aprendizajes, aunque puntualizan que resultaría excesivo decir que la sola presencia de las TIC favorece esta competencia.

El espectro conceptual preponderante corresponde al tradicionalista, resultado que coincide con lo reportado por Urzúa y Rodríguez (2015), quienes declaran que las visiones epistemológicas predominantes en estudiantes universitarios de microbiología son positivistas. La cantidad de respuestas posibilita identificar algunos componentes de los perfiles epistemológico, de aprendizaje y de uso de TIC como se mostrará a continuación:

a) Respecto a las concepciones epistemológicas, destacamos el origen del conocimiento en un contexto delaboratorio, pues desde una perspectiva positivista o empirista el conocimiento proviene de la experimentación, donde las prácticas de laboratorio tienen como objetivo comprobar teorías y desarrollar habilidades manuales, producto de la repetición continua de los procedimientos, es decir, el aprendizaje memorístico de secuencias algorítmicas.

b) En cuanto al aprendizaje, se encuentra un estudiante pasivo que únicamente recibe la información verbal o en forma de materiales que el docente le proporciona, ya que el docente es transmisor de información. Además, se evalúan contenidos declarativos como conceptos, así como la memorización y ejecución de técnicas con secuencias algorítmicas, lo que se relaciona con la perspectiva epistemológica mencionada antes.

c) Finalmente, respecto al uso de TIC, estas son vistas como fuentes de información alternas a los medios tradicionales. El estudiante, como agente pasivo únicamente recibe la información transmitida por el profesor mediante las tecnologías, o bien, busca más por su cuenta con el propósito de acumular una gran cantidad de información, aunque esta no sea procesada. En contraparte, el docente es un transmisor de conocimientos, ya sea mediante la exposición apoyada en los medios tecnológicos o con el material didáctico que él seleccionó. De esta forma, la finalidad de uso de las TIC es proporcionar al alumno la información necesaria para su aprendizaje.

Un ejemplo de la forma en que el espectro conceptual tradicionalista está presente en los estudiantes que participaron, se aprecia en las siguientes expresiones, donde destaca la importancia de un laboratorio para practicar procedimientos y desarrollar habilidades manuales, mientras se dejan de lado los procesos cognitivos superiores.

- E34: en el caso del laboratorio, aún falta práctica - experimental-.

- E35: porque al ser una materia experimental y tomar clases en línea no se logra adquirir las habilidades de trabajo experimental que se realiza en el laboratorio.

- E36: lo que más perjudica fue la parte experimental, en relación con las habilidades/destrezas manuales que estas nos proporcionan. 
Los comentarios del alumnado respecto a la función del profesor en las clases en línea dejan entrever su postura pasiva, pues, desde su perspectiva, es el docente quien debe "impartir la clase" y proporcionarles toda la información y materiales de apoyo para su aprendizaje, lo cual confirma la visión del docente como mero transmisor del conocimiento, ejemplo de ello tenemos:

- E37: el profesor trató de dar los temas lo más entendible posible, y el material adecuado para poder comprender los temas.

- E38: porque nos dieron la información necesaria para entender el porqué de cada cosa.

- E39: porque los profesores siempre dieron su clase, cumpliendo con el horario y proporcionando diferentes materiales de apoyo, además de que se resolvían las dudas durante las sesiones.

En los argumentos expresados por los estudiantes, identificamos que implícitamente su concepción sobre el uso de las TIC es "proporcionarles" la información que requieren para su aprendizaje, como se aprecia en:

- E40: para poder contar con el material necesario para entender los temas que se tratan en clase.

- E41: porque así podemos tener toda la información cuando la necesitemos.

- E42: en el caso de esta materia había que buscar información para realizar las tareas, sin haber tenido clases, ni ningún tipo de referencia o documento que fuera de apoyo. El tiempo que se utilizaba para realizarlas es aún mayor en comparación con una clase presencial, porque todo el peso recae prácticamente en el alumno.

El último comentario destaca que el docente tenía otra visión de aprendizaje, pero el estudiante expresa su inconformidad porque considera que toda la responsabilidad de su aprendizaje recae en él.

Además, destaca la visión de una comunicación unidireccional, donde el estudiante pregunta y el docente contesta, pero no existe un diálogo o intercambio de ideas:

- E43: se puede estar en comunicación con los profesores en cualquier momento, así como el material de trabajo y las tareas que se entregan. Considero que ayudan a tener una buena comunicación por lo que ayuda en las materias

En este sentido, Medina et al. (2020) mencionan que, en la enseñanza de las ciencias, actuar e interactuar socialmente con otras personas permite al estudiante hacer construcciones cognitivas.

Finalmente, las TIC son vistas por algunos estudiantes como fuentes de información alternativas a la biblioteca en que se pueden consultar libros - que pueden llevarse al domicilio-, tesis y artículos de manera presencial. Sin embargo, como se indicó antes, lo importante es acumular información más que su análisis, síntesis y construcción, lo que se evidencia así:

- E44: a pesar de tener información al alcance, siento que no estamos cien por ciento listos para discriminar qué información es correcta y cuál no.

- E45: para buscar información es más fácil que buscar en la biblioteca. 
- E46: tuvimos acceso a mucha bibliografía gracias a la biblioteca digital de la facultad.

\section{Conclusiones}

El análisis en conjunto de las categorías logro de objetivos, comparación del aprendizaje en clases presenciales vs. no presenciales y comparación de la forma de evaluar en clases presenciales y no presenciales, alude a que la mayoría de los argumentos proporcionados por los estudiantes indican que no lograron alcanzar los objetivos de la asignatura, que su aprendizaje fue menor en ERE y que la evaluación fue diferente destacando su preocupación por no haber aprendido las técnicas de laboratorio o por la falta de práctica. Aunque vale la pena señalar que los objetivos de la asignatura únicamente señalan los contenidos relacionados con las habilidades manuales a adquirir, pero deja de lado capacidades importantes que debe formarse en un profesional de las ciencias experimentales y que corresponden a la integración e interpretación de los resultados y a la toma de decisiones que deriva de ello. En este sentido, ya Urzúa (2019) había reportado que el uso de los videos con fines de enseñanza dependía de las concepciones del profesorado sobre el aprendizaje y la NOS, por lo que se considera importante brindar capacitación a los docentes para utilizar las TIC de manera más eficiente con fines de enseñanza. Sin embargo, si sus concepciones se mantienen en un positivismo epistemológico, cercanas al asociacionismo y con perfil técnico transmisor, difícilmente se observará un cambio en la enseñanza de las ciencias, lo que sin duda repercutirá en el estudiantado.

El que la mayoría de los estudiantes aluda al aprendizaje y evaluación de "contenidos declarativos" como lo central en la asignatura evidencia la preocupación por los conceptos y por lo mencionado al inicio de este texto: "trasladar la lógica de las disciplinas científicas a la escuela" (Tamayo, 2009, p. 34), lo que manifiesta un desconocimiento sobre una enseñanza de las ciencias que promueva una actividad científica para pensar, hablar e intervenir en el mundo (Izquierdo, 2005), lo cual implica la posesión de un cuerpo conceptual de conocimientos, un modo para producir el conocimiento - que incluye la observación, experimentación, etc. - y la capacidad para vincular los saberes que se reflejen en actitudes investigativas. Así la intervención experimental adquiere otro sentido, alejado de la visión empíricopositivista (Adúriz-Bravo et al., 2006; Izquierdo et al. 1999; Rodríguez, 2007).

Respecto a la categoría Impacto de la comunicación, si bien la mayoría de los estudiantes destaca que es buena o muy buena la comunicación con los docentes, quienes indican que fue muy mala, comentan que el problema es que es el docente quien no se comunica con ellos, lo cual pone nuevamente la responsabilidad en el docente. Por otro lado, en cuanto a las dos subcategorías del impacto de la comunicación, se presentan algunos comentarios que denotan que una buena comunicación con el docente va acompañada de la utilidad de la asincronía del proceso comunicativo mediado por las TIC.

Más allá de las problemáticas enfrentadas por el estudiantado en ERE, destaca la importancia de que los docentes las conozcan, pues esto resulta significativo para los alumnos, por lo que es importante reflexionar que este conocimiento sobre el estudiantado no sea únicamente en situaciones como la actual, sino en los diferentes contextos de aula. 
La percepción epistemológica predominante en los estudiantes es la positivista, que parece estar influida por la creencia de que una asignatura experimental tiene como único fin el aprendizaje de técnicas con secuencias algorítmicas, dejando de lado procesos cognitivos superiores. Ante ello, se manifiesta la necesidad de replantear el objetivo de la asignatura, donde haya cabida para la formación de procesos cognitivos superiores o de competencias como la interpretación de resultados o evidencias que favorezcan la toma de decisiones, aspectos de suma importancia en la educación superior (Urzúa, 2019).

Es importante destacar que la visión que impera en los estudiantes respecto a sus concepciones epistemológicas, de aprendizaje y uso de TIC es la tradicionalista, lo que implica, por un lado, que los docentes deben actualizarse en sus métodos de aprendizaje y el uso que dan a las TIC. Si bien es cierto que esta situación fue muy particular y faltaron tiempos para una planeación adecuada, también lo es que este mismo hecho fue el que permitió "aflorar" las concepciones de docentes y estudiantes.

Por último, es importante mencionar que, al comparar las concepciones y/o espectros conceptuales de alumnos y docentes entre estudios previos y el presente, es notorio que los estudiantes universitarios poseen el espectro conceptual tradicionalista reflejado en el positivismo, asociacionismo y un enfoque transmisivo-reproductor independientemente del contexto en que se encuentren. Por ello, es importante trabajar para que los estudiantes cambien esta percepción, de forma tal que se vean a sí mismos como sujetos activos responsables de su propio aprendizaje.

\section{Referencias}

Adúriz-Bravo, A. (2008). La naturaleza de la ciencia. En C. Merino, A. Gómez, \& A. AdúrizBravo (Coords.), Área y estrategias de investigación en la didáctica de las ciencias experimentales (pp. 111-125). Universitat Autònoma de Barcelona.

Adúriz-Bravo, A., Salazar, I., Mena, N., \& Badillo, E. (2006). La epistemología en la formación del profesorado de ciencias naturales: aportaciones del positivismo lógico. Revista Electrónica de Investigación en Educación en Ciencias, 1(1), 1-23.

Almirón, M., \& Porro, S. (2014). Las TIC en la enseñanza: un análisis de casos. Revista Electrónica de Investigación Educativa, 16(2), 152-160.

Beltrán, J., Venegas, M., Villar, A., Andrés-Cabello, S., Jareño-Ruiz, D., \& de-GraciaSoriano, P. (2020). Educar en época de confinamiento: La tarea de renovar un mundo común. Revista de Sociología de la Educación, 13(2), 92-104. https://doi.org/10.7203/ RASE.13.2.17187

Blancas, J., \& Rodríguez, D. (2013). Uso de tecnologías en la enseñanza de las ciencias. El caso de una maestra de biología de secundaria. Revista Latinoamericana de Estudios Educativos, 9(1), 162-186.

Canal CODEIC/UNAM. (7 de julio de 2020). Aprender en la contingencia: las experiencias de estudiantes universitarios [Video]. https://www.youtube.com/watch?v=V1TIqOvi9S4

Ernest, P. (1995). The one and the many. En L. Steffe, \& J. Gale (Eds.), Constructivism in Education (pp. 459-586). Lawrence Erlbaum Associates. 
Facultad de Química-UNAM, (julio de 2020). Licenciatura Química Farmacéutico Biológica. https://quimica.unam.mx/ensenanza/licenciaturas/quimica-farmaceutico-biologica/

Habib, H., González, C., Collazos, C., \& Yousef, M. (2020). Estudio exploratorio en Iberoamérica sobre procesos de enseñanza-aprendizaje y propuesta de evaluación en tiempos de pandemia. Education in the Knowledge Society (EKS), 21(17), 1-9. http:// dx.doi.org/10.14201/eks.23537

Izquierdo, M. (2000). Fundamentos epistemológicos. En F. Perales, \& P. Cañal (Dirs.), Didáctica de las ciencias experimentales. Teoría y práctica de la enseñanza de las ciencias (pp. 35-64). Editorial Marfil.

Izquierdo, M. (2005). Hacia una teoría de los contenidos escolares. Enseñanza de las Ciencias, 23(1), 111-122.

Izquierdo, M., Sanmartí, N., \& Espinet, M. (1999). Fundamentación y diseño de las prácticas escolares de ciencias experimentales. Enseñanza de las Ciencias, 17(1), 45-59.

Lederman, N., \& O’Malley, M. (1990). Students' perceptions of tentativeness in science: development, use, and sources of change. Science Education, 74(2), 225-239.

López-Mota, A., Rodríguez, D., \& Bonilla, X. (2004). ¿Cambian los cursos de actualización las representaciones de la ciencia y la práctica docente? Revista Mexicana de Investigación Educativa, 9(22), 699-719.

Marín, N., \& Benarroch, A. (2010). Cuestionario de opciones múltiples para evaluar creencias sobre el aprendizaje de las ciencias. Enseñanza de las Ciencias, 28(2), 245-260.

McComas, W., \& Olson, J. (1998). The nature of science in international science education standards documents. En W. McComas (Ed.), The nature of science in science education. Rationales and strategies (pp. 41-52). Kluger Academic Publishers.

McComas, W., Clough, M., \& Almazroa, H. (1998). The role and Character of the Nature of Science in Science Education. En W. McComas (Ed.), The Nature of Science in Science Education. Rationales and Strategies (pp. 3-39). Kluger Academic Publishers.

Medina, H., Lagunes, A., \& Guerra, T. (2020). ¿Qué aportan las Tecnologías de la Información y Comunicación en la enseñanza de las ciencias? Revista Digital Universitaria, 21(3). http://doi.org/10.22201/codeic.16076079e.2020.v21n3.a9

Mellado, V. (1996). Concepciones y prácticas de aula de profesores de ciencias, en formación inicial de primaria y secundaria. Enseñanza de las Ciencias, 14(3), 289-302.

Mellado, V. (1998). The classroom practice of preservice teachers and their conceptions of teaching and learning science. Science Education, 82, 197-214.

Mellado, V., \& Blanco, L. (2012). Introducción. En V. Mellado, L. Blanco, A. Borrachero, \& J. Cárdenas (Eds.), Las emociones en la enseñanza y el aprendizaje de las ciencias y las matemáticas (pp. VII-XVI). Grupo Deprofe.

Porlán, R. (1988). El pensamiento científico y pedagógico de los maestros en formación. En R. Porlán, J. García, \& P. Cañal (Comps.), Constructivismo y enseñanza de las ciencias (pp. 191-201). Diada Editora. 
Ravanal, E., \& Quintanilla, M. (2012). Concepciones del profesorado de biología en ejercicio sobre el aprendizaje científico escolar. Enseñanza de las Ciencias, 30(2), 33-54. https:// www.raco.cat/index.php/Ensenanza/article/view/254502

Rodrigo, M., \& Cubero, R. (2000). Constructivismo y enseñanza de las ciencias. En F. Perales, \& P. Cañal (Dirs.), Didáctica de las ciencias experimentales. Teoría y práctica de la enseñanza de las ciencias (pp. 85-107). Editorial Marfil.

Rodríguez, D. (2007). Relación entre concepciones epistemológicas y de aprendizaje, con la práctica docente de los profesores de ciencias, a partir de las ideas previas en el ámbito de la física [Tesis Doctoral, Universidad Pedagógica Nacional, México]. Repositorio Institucional Universidad Pedagógica Nacional. http://200.23.113.51/pdf/24355.pdf

Rodríguez, D., \& López-Mota, A. (2006). ¿Cómo se articulan las concepciones epistemológicas y de aprendizaje con la práctica docente en el aula? Tres estudios de caso de profesores de secundaria. Revista Mexicana de Investigación Educativa, 11(31), 1307-1335.

Rowell, J., \& Cawthron, E. (1982). Images of science: an empirical study. European Journal of Science Education, 4(1), 79-94.

Sánchez, M., Martínez, A., Torres, R., Agüero de, M., Hernández, A., Benavides, M., Jaimes, C., \& Rendón, V. (2020). Retos educativos durante la pandemia de covid-19: una encuesta a profesores de la UNAM. Revista Digital Universitaria, 21(3). http://doi.org/10.22201/ codeic.16076079e.2020.v21n3.a12

Solovieva, Y., \& Quintanar, L. (2020). Efectos del confinamiento por la pandemia covid-19 sobre el proceso de enseñanza-aprendizaje en los niveles básicos y medio en el estado de Puebla. Concytep. http://www.concytep.gob.mx/wpcontent/uploads/2020/05/efectos_ del_confinamiento_por_la_pandemia.pdf

Tamayo, O. (2009). Didáctica de las ciencias: la evolución conceptual en la enseñanza y el aprendizaje de las ciencias. Enseñanza de las Ciencias, 17(1), 45-59.

Tsai, C. (2002). A science teacher's reflections and knowledge growth about STS instruction after actual implementation. Science Education, 86(1), 23-41.

Urra, E., Muñoz, A., \& Peña, A. (2013). El análisis del discurso como perspectiva metodológica para investigadores de salud. Enfermería Universitaria, 10(2), 50-57.

Urzúa, M. (2019). La enseñanza de la microbiología mediada por videos: reflexiones de su uso en el laboratorio. En Memorias del $5^{\circ}$ Encuentro universitario de mejores prácticas de uso de TIC en la educación \#educatic2019. UNAM. https://encuentro.educatic.unam. mx/educatic2019/memorias/59.pdf

Urzúa, M., \& Rodríguez, D. (2015). Perfiles conceptuales sobre epistemología, aprendizaje y uso de TIC que guían la práctica educativa de profesores universitarios de ciencias. Memoria Electrónica del XII Congreso Nacional de Investigación Educativa, 2(2),1-8.

Urzúa, M., \& Rodríguez, D. (2016). ¿Qué, para qué, cómo emplean las TIC los docentes universitarios de carreras de química? Anuario Latinoamericano de Educación Química, $31,42-47$ 
Urzúa, M., \& Rodríguez, D. (2017). Perspectiva estudiantil del uso de Moodle para el aprendizaje de las asignaturas experimentales. Revista Digital Universitaria, 18(2), 214

Urzúa, M., Rodríguez, D., \& Martínez, M. (2019). Espectros conceptuales -epistemológico, cognitivo y de uso de TIC- de estudiantes de bachillerato tecnológico en la clase de biología. Bio-Grafía. Escritos sobre la Biología y su Enseñanza, 12(23), 129-139. https:// doi.org/10.17227/bio-grafia.vol.12.num23-9355

\section{Agradecimientos}

A los profesores de la asignatura Microbiología Experimental de la Facultad de Química de la UNAM, que distribuyeron el cuestionario, y a los alumnos que anónima y voluntariamente lo respondieron. 